\title{
Clinical imaging for the prediction of neoadjuvant chemotherapy response in breast cancer
}

\author{
Mitsuhiro Hayashi $^{1,2}$, Yutaka Yamamoto ${ }^{3}$, Hirotaka Iwase $^{2}$ \\ ${ }^{1}$ Department of Breast Surgery, Women's Center, Yotsuya Medical Cube, 7-7 Nibancho, Chiyoda-ku, Tokyo, 102-0084, Japan; ${ }^{2}$ Department of \\ Breast and Endocrine Surgery, Kumamoto City Hospital, 4-1-60 Higashimachi, Higashi-ku, Kumamoto 862-8505, Japan; ${ }^{3}$ Department of Breast and \\ Endocrine Surgery, Graduate School of Medical Sciences, Kumamoto University, 1-1-1 Honjo, Chuo-ku, Kumamoto 860-8556, Japan \\ Contributions: (I) Conception and design: All authors; (II) Administrative support: All authors; (III) Provision of study materials or patients: None; \\ (IV) Collection and assembly of data: All authors; (V) Data analysis and interpretation: All authors; (VI) Manuscript writing: All authors; (VII) Final \\ approval of manuscript: All authors. \\ Correspondence to: Mitsuhiro Hayashi, MD, PhD. Department of Breast Surgery, Women's Center, Yotsuya Medical Cube, 7-7 Nibancho, Chiyoda- \\ ku, Tokyo, 102-0084, Japan. Email: mitsuhayashi-ncd@umin.net.
}

\begin{abstract}
Increased use of cancer screening, improved imaging, and diagnostic intervention techniques has led to the diagnosis of smaller cancers, including breast cancer. Most breast cancer patients receive systemic therapy, and some treatments are given before surgery, such as neoadjuvant therapy, even in an operable setting. Improved neoadjuvant chemotherapy has increased rates of pathological complete response; however, surgery is still required to determine complete tumor remission. Inadequate preoperative evaluations after neoadjuvant therapy can result in excessive surgical stress. Clinical imaging tests such as ultrasound and magnetic resonance imaging of the breast are often performed with neoadjuvant therapy. These clinical imaging techniques, in addition to measuring tumor size, have made it possible to evaluate certain functional aspects of the tumors. Herein, we review the current state of clinical imaging research focused on predicting neoadjuvant chemotherapy response in breast cancer. We also discuss the upfront prediction of treatment response before and during neoadjuvant therapy and the later prediction of pathological residual tumors, including pathological complete response, using ultrasound and magnetic resonance imaging. Upfront prediction can help decision-making and develop new treatment strategies. Predicting the localization of microscopic residual tumors may contribute to disease management without surgery, using radiation or other local treatments. Further larger studies on the prediction of neoadjuvant therapy response using clinical imaging could improve clinical practice and patient benefits.
\end{abstract}

Keywords: Breast cancer; imaging biomarker; prediction; neoadjuvant chemotherapy; elastography; artificial intelligence (AI)

Submitted Mar 16, 2020. Accepted for publication Jun 05, 2020.

doi: $10.21037 /$ cco-20-15

View this article at: http://dx.doi.org/10.21037/cco-20-15

\section{Introduction}

Neoadjuvant therapy refers to the systemic treatment of primary breast cancer before definitive surgical therapy. While all systemic therapies for non-metastatic breast cancer are intended to reduce the risk of recurrence, the purpose of administering neoadjuvant therapy before surgery is to downstage the tumor. This may allow less extensive surgery of the breast and/or ipsilateral axilla, including providing more choices for patients who are able to undergo breast-conserving surgery in place of mastectomy, improved cosmetic outcomes, and reduced postoperative complications such as lymphedema of the arm. Neoadjuvant therapy also permits an early individual evaluation of the effectiveness of a selected systemic therapy. The surrogate endpoint, as reflected by the presence and 
extent or absence of residual tumor cells after neoadjuvant therapy, is a strong prognostic factor for the risk of recurrence, particularly in aggressive types of breast cancer. In addition to these clinical benefits, neoadjuvant therapy provides researchers the opportunity to conduct imaging studies as well as obtain biological specimens before, during, and after the preoperative treatment, which may allow us to identify tumor-specific biomarkers of treatment response or resistance. At present, neoadjuvant therapy has largely taken the form of chemotherapy. Herein, we present a review of imaging studies associated with neoadjuvant chemotherapy (NAC).

Before NAC, ultrasound (US or sonography or echography) of the breast should be performed to document tumor size. Sometimes, magnetic resonance imaging (MRI) may be helpful in calculating tumor volume and extent in the breast and making decisions on whether to undergo breast-conserving surgery, in addition to the diagnostic capability of breast tumor at the time of the first diagnosis. Once a patient has completed NAC, imaging tests should be performed to guide the surgical approach. US imaging of the involved breast and ipsilateral axilla is usually required. Additionally, MRI may be obtained if the tumor is not well visualized on US or if a better definition of the extent of tumor would assist the surgeon in determining the optimal surgical approach depending on the institution. In patients considering breast-conserving therapy, pretreatment or repeated US and MRI have been clinically important to appropriately assess the presence of tumor distribution.

In this study, we describe the current status of these imaging tests, i.e., US and MRI, which are commonly used in the current clinical setting, in predicting the effects of NAC in breast cancer treatment with those basic knowledge. Specifically, we address the upfront prediction of subsequent treatment responsiveness before and during NAC, and prediction of pathological evaluation, or concordance between imaging findings and pathological diagnosis of treatment responsiveness such as pathological complete response (pCR) after NAC, which is useful for surgical decision. Improved systemic therapy, moleculetargeted therapy, and identification of biomarkers have increased rates of pCR after NAC in breast cancer; hence, pCR is achieved in $37-50 \%$ of triple negative breast cancer types [estrogen receptor (ER) negative; progesterone receptor negative; human epidermal growth factor receptor type 2 (HER2) negative status], and in 50-70\% of HER2 type after NAC $(1,2)$. Predicting the effects of treatment before and during NAC can help in making decisions during treatment and developing new molecular therapies by de-escalating long-term treatment procedures. Predicting pathological tumor size after the completion of NAC, particularly in pCR, will help to gain time and avoid surgery. Identification of pCR without surgery, such as a combination of precise assessment of clinical imaging and needle biopsies, may reduce treatment burden. Furthermore, precise detection of the pCR is not important because residual tumor cells will be controlled with nonsurgical local therapy and subsequent systemic therapies. It is, however, necessary to accurately measure tumor burden by improving clinical imaging.

\section{Early prediction of NAC responses by breast US}

US is a dynamic examination in which image generation is based on the conduction and reflection of high-frequency mechanical sound waves in the breast tissue. Information of ultrasonic impulses and their reflections as echoes is converted and processed into a real-time image. Differences in the penetration and reflection of sound waves between a normal and a diseased breast tissue enable diagnostic examination and image scaling. In addition to the basic brightness mode (B-mode) that depends on acoustic impedance and anatomy, there are several techniques that aid in clinical assessment, such as Doppler imaging, elastography, contrast-enhanced imaging, 3D imaging, and automated volume scanning.

In view of the various US techniques used to analyze anatomy and morphology, there are few reports on the upfront prediction of NAC response, except on US elastography. Elastography is a novel technique that can provide additional information that was previously not available. Elastography is an imaging modality based on mechanical properties and tissue stiffness that shows relative differences in stiffness among tissues. Although physicians have used palpation for the diagnosis of breast cancer, as stiffer masses are more likely to be malignancies, elastography has the potential to quantify the stiffness of a lesion. This indicates that elastography could be an excellent technique for differentiating breast diseases. Specifically, there are two types of elastography; strain elastography and shear wave elastography. The strain type produces an image based on the displacement of the tissue from a compression/release force applied by external forces such as transducer compression, patient breathing/heartbeat, or acoustic radiation force impulse (ARFI). The shear wave type applies a push pulse, ARFI, which results in shear wave 
propagation that can be measured as a velocity. Because the velocity of the shear wave through tissues depends on the tissue mechanics, a value of the stiffness can be obtained.

There are only few large-scale studies that have used breast US, and most reports are from single institutions. Since 2012, there have been reports on the relationship between pretreatment evaluation using elastography and subsequent response of breast cancer to NAC. Hayashi et al. first reported that tumor stiffness assessed using strain elastography was related to the proportion of patients with a successful pCR, who receiving NAC (3). They examined the correlation between elasticity score and treatment responses in 55 patients with breast cancer, which showed that the harder tumors had less chance of clinical responses and pCR: hard, $14 \%$ of pCR vs. soft, $50 \%$ of pCR. Multivariate analysis of pCR, including standard biomarkers, showed that tumor elasticity was a significant factor, as well as ER and HER2 status. Their findings were concordant with those of a subsequent study conducted by Evans $e t$ al. who used shear wave elastography (4). They compared pretreatment tumor stiffness, mean $\mathrm{kPa}$, and pathological residual cancer burden (RCB) score and tumor cellularity in 44 patients treated with NAC. Their statistical assessment showed that pretreatment quantitative stiffness had a positive correlation with residual tumor cellularity after NAC, with a correlation coefficient of 0.35 . Although the number of patients with each breast cancer subtype was small, this relationship was seen in the HER2 subgroup analysis. Falou et al. reported that changes in tumor stiffness after NAC were different between responding and non-responding tumors in 15 patients analyzed (5). Their data suggested that the strain ratio obtained 4 weeks after starting NAC was a potential predictor of tumor response when compared with initial stiffness because patients who had pathologic response to NAC, but not patients with non-responsive tumors, showed approximately $20 \%$ decrease in the strain ratio. Jing et al. conducted a prospective study and used shear wave elastography and showed that relative changes in tumor stiffness after two cycles of NAC correlated significantly with pathological responses of the postoperative specimens, in addition to baseline mean tumor stiffness: $82.8 \mathrm{kPa}$ in responders vs. $99.8 \mathrm{kPa}$ in non-responders (6). They reported that the threshold of changes in stiffness after two cycles of NAC, distinguishing responders and nonresponders, was $-36.1 \%$, with $72.9 \%$ sensitivity and $85.7 \%$ specificity. Ma et al. used both strain and shear wave types and reported that the relative changes in the elastography score after two cycles of NAC had the potential to predict
NAC responses in 71 patients in a prospective analysis (7). They also suggested that adding the immunohistochemical proliferative score, namely, the Ki-67 index, to elastography results may improve the predictive power for responses to NAC (8). Evans et al. also proposed that shear wave stiffness of $>50 \mathrm{kPa}$ after three cycles of NAC and relative changes from baseline to three cycles had a strong potential to predict pCR after completion of NAC (9). Similarly, recent reports published by Katyan et al., Fang et al., and Fernandes et al. showed that early reduction in tumor stiffness after two or three cycles of NAC had a predicting potential for better response of subsequent treatment (10-12). Taken together, baseline tumor stiffness evaluated by US elastography and its early reactivity may predict subsequent responses to NAC. At present, no reports of intervention trials that change the treatment strategy for breast cancer depending on the stiffness have been documented. Progress in the standardization of measurement, multi-center study, and biological research that elucidates the tumor stiffness mechanism would be required to provide any clinical benefits.

As Doppler US is now assumed to be an essential technique in US examination, monitoring tumor response using Doppler imaging has also been applied during any treatment (13-15). Doppler US can visualize realtime flow movements as colored areas in a defined image of the B-mode. Measurement of tissue perfusion can be helpful in differentiating between benign and malignant tumors because growing tumors usually have increased vascularization. The development of the color or power Doppler technique has made it possible to detect small vessels and slow flows. Kumar et al. analyzed 50 patients and reported that tumor with higher Doppler characteristics such as high flow velocity and the number of flow signals tended to achieve complete response (16). They calculated the Doppler scores using peak flow velocity, peak systolic velocity, and the number of flow signals and compared them with the histological response after chemotherapy. Kuo et al. showed that early vascularity change after neoadjuvant chemotherapy rather than initial high vascularity was correlated with final treatment responses in 30 patients with locally advanced breast cancer (17). In relation to Doppler US, contrast-enhanced US technique has been used to monitor the NAC response (18). After 20 to 40 seconds of intravenous injection, the contrast agent reaches the article vessels and produces improved visualization of the vessels. While contrast-enhanced US increase the sonographic reflectivity of blood and aids in diagnostic differentiation in 
certain cases, the predictive value is still unexcavated.

Three-dimensional (3D) US is based on the acquisition and processing of numerous closely spaced two-dimensional image sections. A dedicated probe affords automatic guidance of the transducer and ensures a geometrically correct $3 \mathrm{D}$ reconstruction. $3 \mathrm{D}$ visualization of breast tumors can facilitate spatial presentation, image interpretation, and volume calculation. Gounaris et al. performed 3D volumetric US measurements before, in the middle, and at the end of NAC in a subset of 55 patients from the Neo-tAnGo trial (19). They reported that residual tumor volume after four cycles of NAC was likely to be predictive of pCR, while tumor size changes based on the longest diameter were not predictive. In addition, the combination approach between $3 \mathrm{D}$ reconstruction and functional assessment has increased. Shia et al. reported that 3D power Doppler US was able to predict pCR with an accuracy of $93.1 \%$ and a specificity of $85.5 \%$ with the measurements conducted after the first cycle of NAC in 29 patients with T2 stage breast cancer (20). Athanasiou et al. reported that reduction of tumor elasticity and heterogeneity during NAC measured using 3D elastography was correlated with a good response (21). In addition to US elastography, several US techniques have been used for clinical assessment during NAC. These studies have a small sample size, often a retrospective analysis, which supports the need for further validation to determine whether these are true observations. These exploratory studies can help plan the subsequent trials to improve clinical utility.

\section{Concordance with pathological diagnosis on breast US measurement}

After completion of NAC regimens, if we accurately predict the residual pathological tumor distribution in a specimen through a preoperative imaging test, surgical planning including breast-conserving surgery can be optimized and finally may be omitted in certain patients, such as those who achieved pCR. Practically, B-mode of US has been used to assess tumor size in breast cancer patients after NAC in addition to physical examination (22-26). In the early 2000s, Chagpar et al. reported that the size estimate of residual tumor by US was moderately correlated $(\rho=0.42)$ with pathological assessment after eight cycles of paclitaxel and anthracycline-based NAC in 189 patients. The ability to predict greater tumor dimension within $1 \mathrm{~cm}$ compared with pathological tumor size was $75 \%$ in US, $66 \%$ in clinical examination, and $70 \%$ in mammography (24). Peintinger et al. reported that the combination of mammography and US had a high accuracy in predicting pCR after NAC, accuracy $88.9 \%$, sensitivity $78.6 \%$, and specificity $92.5 \%$, in 162 patients, including 24 patients with pCR (24). While preoperative measurement using US will continue to play an important role due to consecutive improvements in the basic performance of the echo device, lack of concordance between conventional clinical test and pathological evaluation of NAC response remains a subject of debate. This may be caused by the variable patterns of tumor response to NAC, which ranges from symmetric shrinkage around a central core, concentric pattern, to complete resolution of a discrete mass despite persistence of microscopic foci of residual tumor cells, multicentric or dendritic shrinkage patterns. To increase the diagnostic capability of pre-surgery imaging tests after NAC, several US techniques have been tried.

Using Doppler US, Roubidoux et al. reported that US yielded false-negative results in detecting tumors smaller than $6 \mathrm{~mm}$ in a study of 34 patients treated with NAC (27). After NAC with doxorubicin and docetaxel, tumor vascularity usually decreased, but this was not specific for pCR. They showed three false-positive and four falsenegative cases using the Doppler US study. Vallone et al. reported that addition of a contrast agent to Doppler US was able to increase the sensitivity of residual tumor detection after NAC in 50 locally advanced patients (28). Wang et al. reported that the combination of contrastenhanced and elastography US improved the accuracy of NAC responses in 65 breast cancer studies (29). Lee et al. designed a study with 71 stage II-III patients to evaluate the improvement of residual tumor detection using shearwave elastography US (30). The sensitivity, specificity, and accuracy of elastography were improved to $83.6 \%, 80.0 \%$, and $83.1 \%$, respectively, compared to those of B-mode US, when a maximum elasticity value of $>30 \mathrm{kPa}$ considered to indicate the presence of residual cancer. A combined assessment of B-mode and elastography, comparable to MRI, was proposed. Athanasiou et al. reported a feasibility study of 33 patients and reported that 3D US and 3D shearwave elastographies were comparable to contrasted MRI for measuring residual tumor volume (21). Lee et al. reported that preoperative estimation of residual tumor volume or pCR by B-mode and 3D US was similar to MRI assessment in a study of 42 patients (31). Jia et al. reported the 3D contrastenhanced US evaluation in a study of 48 patients (32). Pretreatment evaluation correlated well with tumor microvessel density using biopsy immunohistochemistry 
and preoperative evaluation, and the changes after NAC were significantly different between pCR and non-PCR tumors.

US can be expected to have further clinical applications in various approaches due to its excellent convenience. Morphological measurements of small residual disease by B-mode US may occasionally be difficult to distinguish scar formation after NAC and residual active tumor. Adding any functional US techniques may help in diagnosing whether there is truly a pCR or a residual active tumor. In the next step, following these previous findings, prospective multicenter trials with appropriate sample sizes targeting unresolved points will be required in the US fields. These developments can promote the disappearance of invasive tumor cells, although residual tumor cells in the ducts may be difficult, which may contribute to the option of omitting surgery and proceed with local treatment such as radiation therapy.

\section{Early prediction of NAC responses using breast MRI}

MRI of the breast has high sensitivity for the detection of hypervascularized areas in the breast, such as in breast cancer, by depicting the intermammary structures before and after the administration of a contrast agent. Breast MRI should be performed with field strengths of 1.5 and 3.0 Tesla. Spatial bilateral surface coils are required to achieve a sufficiently high spatial resolution. Contrast agents enhance the areas that characterized by increased vascularization, increased permeability, and expanded interstitial matrix spaces. These characteristics are found in almost all malignant tumors; however, some benign diseases also show these increased perfusion patterns. Therefore, there are some additional techniques to diagnose the overlap in imaging characteristics of malignant and benign lesions, such as time-signal intensity curves and diffusion-weighted MRI (DWI). Curve analysis in the targeted areas of contrast enhancement has been implicated in differentiating invasive cancer, noninvasive cancer, and benign lesions based on contrast patterns such as rapid, plateau, and washout kinetics. DWI is a noninvasive imaging technique that assesses the diffusional movement of water molecules in the tissue, based on the observation that water diffusion is abnormal in breast cancers. This technique is used primarily to examine the brain diseases such as strokes. The signal strength of water molecules is proportional to the degree of diffusion. The results of DWI are calculated and then reported with a quantitative measure, which is termed the apparent diffusion coefficient (ADC), for the tissue studied. Breast cancers demonstrate restricted or lower water diffusion than do normal breast tissue or benign breast lesions, which results in a lower ADC.

Pickles et al. reported that early changes in the dynamic contrast-enhancement parameters of MRI were different between 48 responders and 20 non-responders to NAC (33). Martincich et al. also reported that the reductions in tumor volume and early enhancement ratio after two cycles of NAC showed a better predictive value for $\mathrm{pCR}$ in 30 patients with breast cancer (34). A prospective multicenter trial sponsored by the American College of Radiology Imaging Network (ACRIN) study 6698 was completed to determine whether the change in tumor DW-ADC on MRI predicts pCR to NAC in 272 consecutive women with breast cancer (35). They reported that changes in tumor $\mathrm{ADC}$ at mid-treatment, after 12 weeks, from the baseline were more predictive [area under the curves (AUC) 0.72, 95\% confidence interval (CI): 0.61-0.83] for pCR after NAC. As large-scale research on MRI has been carried out, it is expected to have a certain early prediction ability of pCR. Within breast cancer subtypes in which pCR is assumed to be a prognostic surrogate factor, MRI in the early prediction of NAC responses may be a promising tool for treatment change and drug development.

\section{Concordance with pathological diagnosis on breast MRI measurement}

More research has been performed to compare MRI tumor assessment after NAC completion, before surgery, with pathological tumor distribution, compared to US-based studies. Cheung et al. reported that residual tumor size on MRI correlated well with microscopic tumor size in a study of 33 patients (36). Choi et al. proposed that additional factors including tumor distribution pattern, background parenchymal enhancement pattern, and kinetic curve pattern as well as tumor size were helpful in increasing the diagnostic accuracy of MRI for evaluating residual disease after NAC (37). Vriens et al. reported that MRI estimated residual tumor size with $<10 \mathrm{~mm}$ discordance in $54 \%$ of patients, overestimated size in $28 \%$, and underestimated size in $18 \%$ of patients in a study of 182 locally advanced breast cancer patients from the INTENS clinical trial (38). They studied the results of both MRI and US, which indicated a similar ability of these two approaches to accurately measure the size of residual tumor after NAC with US estimated 
residual tumor size with $<10 \mathrm{~mm}$ discordance in $63 \%$ of patients, overestimated size in $20 \%$, and underestimated size in $17 \%$ of patients, respectively. Marinovich et al. performed a meta-analysis of 19 studies including a total of 958 patients and showed that MRI appeared to slightly overestimate pathologic tumor size, but measurement errors may be large enough to be clinically significant (39). They reported that agreement with pathology was better for MRI and US measurement. Similar to concordance with pathological tumor diagnosis using MRI, the prediction of pCR by MRI has also been studies. Schott et al. reported that the accuracy of physical examination, $M M G$, US, and MRI in determining pCR was $75 \%, 89 \%, 82 \%$, and $89 \%$, respectively in 43 patients in a study involving doxorubicin and docetaxel for four cycles (40). Yuan et al. performed a meta-analysis of $25 \mathrm{NAC}$ studies that accumulated a total of 1,212 patients and showed that contrast-enhanced MRI had high specificity $(91 \%)$ in predicting pCR, while the sensitivity was relatively low (63\%) (41). A prospective multicenter trial sponsored by the ACRIN study 6657 studied the accuracy of preoperative measurements for detecting the disease after NAC in 138 patients with $>3 \mathrm{~cm}$ breast cancer (42). Assessment of the longest diameter by MRI showed higher accuracy than that by clinical examination and MMG. There was little difference across preoperative measurements in the accuracy of detecting pCR particularly for single masses (AUC $=0.69-0.72$ ). The prediction of pathological tumor spread using MRI after completion of NAC has a certain consistency as in the US assessment. Further research focusing on breast cancer subtypes or treatment regimens is warranted, which needs to be understood with a clear purpose, whether to predict pCR or residual tumor size.

\section{Perspectives and limitations}

Technological advances in clinical imaging hold promise in addressing such challenges across the spectrum of cancer detection, characterization, treatment, and monitoring. The interpretation of the big data generated by these advancements can present a new medical innovation. Recent advances in artificial intelligence (AI) have made great strides in automatically quantifying radiographic patterns in medical imaging data. Deep learning, a subset of $\mathrm{AI}$, is an especially promising approach that automatically learns feature representations from sample images and has been shown to match human performance in task-specific applications (43). Breast cancer screening is certainly an ideal application for $\mathrm{AI}$ in medical imaging because large well-curated datasets suitable for algorithm training and testing are already available. Recently, McKinney et al. reported that deep learning-based AI was able to identify breast cancer more accurately than radiologists (44). They trained their AI algorithm with mammograms from 25,856 women in the United Kingdom and 3,097 women in the United States and then used these mammograms to identify the presence of breast cancer in the mammograms of women who were known to have had either biopsy-proven breast cancer or normal follow-up imaging results at least one year later. They reported that the AI system outperformed both historical decisions made by the radiologists who initially assessed the mammograms and the decisions of six expert radiologists who interpreted 500 randomly selected cases in a controlled study. Their algorithm led to an absolute reduction of $5.7 \%$ in false-positive findings for the US and that of $1.2 \%$ for the UK dataset and a reduction of $9.4 \%$ and $2.7 \%$ in false-negative findings, respectively.

However, AI systems are tools still under development because the real world is more complicated and diverse than the type of controlled research environment. For instance, most images of previous AI studies were obtained using a mammography system from a single manufacturer. Patient demographics in clinical practice are of great variety. AI studies may need to learn from an earlier computer system, computer-aided detection (CAD), which has not resulted in a significant change in specificity and has not been speculated to be useful in practice as experimental data (45). Clinical trials will be required to further assess the utility of the AI system in clinical imaging. The AI system may have a role in aiding the early detection of breast cancer and hopefully predict treatment responses. Not only AI researchers but also imaging biomarker researchers will be needed to consider multiple steps and goals, including grant support and governmental approval for medical devices to advance clinical imaging studies.

\section{Conclusions}

The goals of NAC were to achieve operability, enable breast conservation, and prognostic information historically. The evolving purpose has been to individualize therapy based on the response, investigate novel therapies, and identify biomarkers of the response. To accelerate achieving these goals, imaging analysis must be involved. Certain imaging tests can evaluate not only the measurement of tumor distribution but also the characteristics of the tumor such as 
imaging biomarkers. For instance, it has been reported that imaging characteristics, including those obtained on MRI, were closely associated with tumor molecular subtypes $(46,47)$. In addition, functional imaging analysis showed the prognostic ability of functional tumor volume measured by MRI as a predictor of patient recurrence who received NAC in ACRIN and I-SPY1 trials (48). The use of AI in cancer imaging research has already started. Funding agencies should support more aggressively, as it is not a well-funded research area, unlike the field of drug development. The cross-link study of clinical imaging and drug responses will contribute to a wide range of clinical applications and patient benefits.

\section{Acknowledgments}

We thank the editors, Dr. Yutaka Yamamoto and Dr. Takayuki Ueno, and all the people involved in this study for helpful discussions.

Funding: None.

\section{Footnote}

Provenance and Peer Review: This article was commissioned by the Guest Editors (Yutaka Yamamoto and Takayuki Ueno) for the series "Neoadjuvant/Adjuvant Treatment for Early Breast Cancer" published in Chinese Clinical Oncology. The article was sent for external peer review organized by the Guest Editors and the editorial office.

Conflicts of Interest: All authors have completed the ICMJE uniform disclosure form (available at http://dx.doi. org/10.21037/cco-20-15). The series "Neoadjuvant/Adjuvant Treatment for Early Breast Cancer" was commissioned by the editorial office without any funding or sponsorship. YY served as the unpaid Guest Editor of the series, and serves as an unpaid editorial board member of Chinese Clinical Oncology from May 2019 to April 2021. The other authors have no other conflicts of interest to declare.

Ethical Statement: The authors are accountable for all aspects of the work in ensuring that questions related to the accuracy or integrity of any part of the work are appropriately investigated and resolved.

Open Access Statement: This is an Open Access article distributed in accordance with the Creative Commons Attribution-NonCommercial-NoDerivs 4.0 International
License (CC BY-NC-ND 4.0), which permits the noncommercial replication and distribution of the article with the strict proviso that no changes or edits are made and the original work is properly cited (including links to both the formal publication through the relevant DOI and the license). See: https://creativecommons.org/licenses/by-nc-nd/4.0/.

\section{References}

1. Wuerstlein R, Harbeck N. Neoadjuvant Therapy for HER2-positive Breast Cancer. Rev Recent Clin Trials 2017;12:81-92.

2. Poggio F, Bruzzone $M$, Ceppi $M$, et al. Platinum-based neoadjuvant chemotherapy in triple-negative breast cancer: a systematic review and meta-analysis. Ann Oncol 2018;29:1497-508.

3. Hayashi M, Yamamoto Y, Ibusuki M, et al. Evaluation of tumor stiffness by elastography is predictive for pathologic complete response to neoadjuvant chemotherapy in patients with breast cancer. Ann Surg Oncol 2012;19:3042-9.

4. Evans A, Armstrong S, Whelehan P, et al. Can shearwave elastography predict response to neoadjuvant chemotherapy in women with invasive breast cancer? $\mathrm{Br} \mathrm{J}$ Cancer 2013;109:2798-802.

5. Falou O, Sadeghi-Naini A, Prematilake S, et al. Evaluation of neoadjuvant chemotherapy response in women with locally advanced breast cancer using ultrasound elastography. Transl Oncol 2013;6:17-24.

6. Jing H, Cheng W, Li ZY, et al. Early Evaluation of Relative Changes in Tumor Stiffness by Shear Wave Elastography Predicts the Response to Neoadjuvant Chemotherapy in Patients With Breast Cancer. J Ultrasound Med 2016;35:1619-27.

7. Ma Y, Zhang S, Li J, et al. Comparison of strain and shear-wave ultrasounic elastography in predicting the pathological response to neoadjuvant chemotherapy in breast cancers. Eur Radiol 2017;27:2282-91.

8. Ma Y, Zhang S, Zang L, et al. Combination of shear wave elastography and $\mathrm{Ki}-67$ index as a novel predictive modality for the pathological response to neoadjuvant chemotherapy in patients with invasive breast cancer. Eur J Cancer 2016;69:86-101.

9. Evans A, Whelehan P, Thompson A, et al. Prediction of Pathological Complete Response to Neoadjuvant Chemotherapy for Primary Breast Cancer Comparing Interim Ultrasound, Shear Wave Elastography and MRI. Ultraschall Med 2018;39:422-31. 
10. Fang C, Yang T. Value of tissue elastography in the prediction of efficacy of neoadjuvant chemotherapy in breast cancer. J BUON 2019;24:555-9.

11. Fernandes J, Sannachi L, Tran WT, et al. Monitoring Breast Cancer Response to Neoadjuvant Chemotherapy Using Ultrasound Strain Elastography. Transl Oncol 2019;12:1177-84.

12. Katyan A, Mittal MK, Mani C, et al. Strain wave elastography in response assessment to neo-adjuvant chemotherapy in patients with locally advanced breast cancer. Br J Radiol 2019;92:20180515.

13. Lagalla R, Caruso G, Finazzo M. Monitoring treatment response with color and power Doppler. Eur J Radiol 1998;27 Suppl 2:S149-56.

14. Huber S, Medl M, Helbich T, et al. Locally advanced breast carcinoma: computer assisted semiquantitative analysis of color Doppler ultrasonography in the evaluation of tumor response to neoadjuvant chemotherapy (work in progress). J Ultrasound Med 2000;19:601-7.

15. Singh S, Pradhan S, Shukla RC, et al. Color Doppler ultrasound as an objective assessment tool for chemotherapeutic response in advanced breast cancer. Breast Cancer 2005;12:45-51.

16. Kumar A, Singh S, Pradhan S, et al. Doppler ultrasound scoring to predict chemotherapeutic response in advanced breast cancer. World J Surg Oncol 2007;5:99.

17. Kuo WH, Chen CN, Hsieh FJ, et al. Vascularity change and tumor response to neoadjuvant chemotherapy for advanced breast cancer. Ultrasound Med Biol 2008;34:857-66.

18. Cao X, Xue J, Zhao B. Potential application value of contrast-enhanced ultrasound in neoadjuvant chemotherapy of breast cancer. Ultrasound Med Biol 2012;38:2065-71.

19. Gounaris I, Provenzano E, Vallier AL, et al. Accuracy of unidimensional and volumetric ultrasound measurements in predicting good pathological response to neoadjuvant chemotherapy in breast cancer patients. Breast Cancer Res Treat 2011;127:459-69.

20. Shia WC, Chen DR, Huang YL, et al. Effectiveness of evaluating tumor vascularization using $3 \mathrm{D}$ power Doppler ultrasound with high-definition flow technology in the prediction of the response to neoadjuvant chemotherapy for T2 breast cancer: a preliminary report. Phys Med Biol 2015;60:7763-78.

21. Athanasiou A, Latorre-Ossa H, Criton A, et al. Feasibility of Imaging and Treatment Monitoring of Breast Lesions with Three-Dimensional Shear Wave Elastography.
Ultraschall Med 2017;38:51-9.

22. Fiorentino C, Berruti A, Bottini A, et al. Accuracy of mammography and echography versus clinical palpation in the assessment of response to primary chemotherapy in breast cancer patients with operable disease. Breast Cancer Res Treat 2001;69:143-51.

23. Chagpar AB, Middleton LP, Sahin AA, et al. Accuracy of physical examination, ultrasonography, and mammography in predicting residual pathologic tumor size in patients treated with neoadjuvant chemotherapy. Ann Surg 2006;243:257-64.

24. Peintinger F, Kuerer HM, Anderson K, et al. Accuracy of the combination of mammography and sonography in predicting tumor response in breast cancer patients after neoadjuvant chemotherapy. Ann Surg Oncol 2006;13:1443-9.

25. Keune JD, Jeffe DB, Schootman M, et al. Accuracy of ultrasonography and mammography in predicting pathologic response after neoadjuvant chemotherapy for breast cancer. Am J Surg 2010;199:477-84.

26. Croshaw R, Shapiro-Wright H, Svensson E, et al. Accuracy of clinical examination, digital mammogram, ultrasound, and MRI in determining postneoadjuvant pathologic tumor response in operable breast cancer patients. Ann Surg Oncol 2011;18:3160-3.

27. Roubidoux MA, LeCarpentier GL, Fowlkes JB, et al. Sonographic evaluation of early-stage breast cancers that undergo neoadjuvant chemotherapy. J Ultrasound Med 2005;24:885-95.

28. Vallone P, D'Angelo R, Filice S, et al. Color-doppler using contrast medium in evaluating the response to neoadjuvant treatment in patients with locally advanced breast carcinoma. Anticancer Res 2005;25:595-9.

29. Wang B, Jiang T, Huang M, et al. Evaluation of the response of breast cancer patients to neoadjuvant chemotherapy by combined contrast-enhanced ultrasonography and ultrasound elastography. Exp Ther Med 2019;17:3655-63.

30. Lee SH, Chang JM, Han W, et al. Shear-Wave Elastography for the Detection of Residual Breast Cancer After Neoadjuvant Chemotherapy. Ann Surg Oncol 2015;22 Suppl 3:S376-84.

31. Lee MC, Gonzalez SJ, Lin H, et al. Prospective trial of breast MRI versus 2D and 3D ultrasound for evaluation of response to neoadjuvant chemotherapy. Ann Surg Oncol 2015;22:2888-94.

32. Jia WR, Tang L, Wang DB, et al. Three-dimensional Contrast-enhanced Ultrasound in Response Assessment 
for Breast Cancer: A Comparison with Dynamic Contrastenhanced Magnetic Resonance Imaging and Pathology. Sci Rep 2016;6:33832.

33. Pickles MD, Lowry M, Manton DJ, et al. Role of dynamic contrast enhanced MRI in monitoring early response of locally advanced breast cancer to neoadjuvant chemotherapy. Breast Cancer Res Treat 2005;91:1-10.

34. Martincich L, Montemurro F, De Rosa G, et al. Monitoring response to primary chemotherapy in breast cancer using dynamic contrast-enhanced magnetic resonance imaging. Breast Cancer Res Treat 2004;83:67-76.

35. Partridge SC, Zhang Z, Newitt DC, et al. Diffusionweighted MRI Findings Predict Pathologic Response in Neoadjuvant Treatment of Breast Cancer: The ACRIN 6698 Multicenter Trial. Radiology 2018;289:618-27.

36. Cheung YC, Chen SC, Su MY, et al. Monitoring the size and response of locally advanced breast cancers to neoadjuvant chemotherapy (weekly paclitaxel and epirubicin) with serial enhanced MRI. Breast Cancer Res Treat 2003;78:51-8.

37. Choi BB, Kim SH. Effective factors to raise diagnostic performance of breast MRI for diagnosing pathologic complete response in breast cancer patients after neoadjuvant chemotherapy. Acta Radiol 2015;56:790-7.

38. Vriens BE, de Vries B, Lobbes MB, et al. Ultrasound is at least as good as magnetic resonance imaging in predicting tumour size post-neoadjuvant chemotherapy in breast cancer. Eur J Cancer 2016;52:67-76.

39. Marinovich ML, Macaskill P, Irwig L, et al. Metaanalysis of agreement between MRI and pathologic breast tumour size after neoadjuvant chemotherapy. Br J Cancer 2013;109:1528-36.

40. Schott AF, Roubidoux MA, Helvie MA, et al. Clinical and radiologic assessments to predict breast cancer pathologic

Cite this article as: Hayashi M, Yamamoto $\mathrm{Y}$, Iwase H. Clinical imaging for the prediction of neoadjuvant chemotherapy response in breast cancer. Chin Clin Oncol 2020;9(3):31. doi: 10.21037/cco-20-15 complete response to neoadjuvant chemotherapy. Breast Cancer Res Treat 2005;92:231-8.

41. Yuan Y, Chen XS, Liu SY, et al. Accuracy of MRI in prediction of pathologic complete remission in breast cancer after preoperative therapy: a meta-analysis. AJR Am J Roentgenol 2010;195:260-8.

42. Scheel JR, Kim E, Partridge SC, et al. MRI, Clinical Examination, and Mammography for Preoperative Assessment of Residual Disease and Pathologic Complete Response After Neoadjuvant Chemotherapy for Breast Cancer: ACRIN 6657 Trial. AJR Am J Roentgenol 2018;210:1376-85.

43. Bi WL, Hosny A, Schabath MB, et al. Artificial intelligence in cancer imaging: Clinical challenges and applications. CA Cancer J Clin 2019;69:127-57.

44. McKinney SM, Sieniek M, Godbole V, et al. International evaluation of an AI system for breast cancer screening. Nature 2020;577:89-94.

45. LeCun Y, Bengio Y, Hinton G. Deep learning. Nature 2015;521:436-44.

46. Zhu Y, Li H, Guo W, et al. Deciphering Genomic Underpinnings of Quantitative MRI-based Radiomic Phenotypes of Invasive Breast Carcinoma. Sci Rep 2015;5:17787.

47. Li H, Zhu Y, Burnside ES, et al. MR Imaging Radiomics Signatures for Predicting the Risk of Breast Cancer Recurrence as Given by Research Versions of MammaPrint, Oncotype DX, and PAM50 Gene Assays. Radiology 2016;281:382-91.

48. Hylton NM, Gatsonis CA, Rosen MA, et al. Neoadjuvant Chemotherapy for Breast Cancer: Functional Tumor Volume by MR Imaging Predicts Recurrence-free Survival-Results from the ACRIN 6657/CALGB 150007 I-SPY 1 TRIAL. Radiology 2016;279:44-55. 